\title{
Vie et mort de deux femmes juives. À l'ombre d'un mari et d'un père
}

Danielle Delmaire et Jean-Michel Faidit

\section{(2) OpenEdition}

1 Journals

Édition électronique

URL : https://journals.openedition.org/tsafon/405

DOI : $10.4000 /$ tsafon.405

ISSN : 2609-6420

Éditeur

Association Jean-Marie Delmaire

Édition imprimée

Date de publication : 1 décembre 2017

Pagination : 105-130

ISSN : 1149-6630

\section{Référence électronique}

Danielle Delmaire et Jean-Michel Faidit, «Vie et mort de deux femmes juives. À l'ombre d'un mari et d'un père », Tsafon [En ligne], 74 | 2017, mis en ligne le 31 mai 2018, consulté le 23 juin 2021. URL : http://journals.openedition.org/tsafon/405 ; DOI : https://doi.org/10.4000/tsafon.405 


\title{
Varia : Histoire
}

\section{Vie et mort de deux femmes juives À l'ombre d'un mari et d'un père}

\author{
Danielle Delmaire* \\ Jean-Michel Faidit ${ }^{* *}$
}

La ville de Lille honore le médecin Désiré Verhaeghe (1874-1927) en lui attribuant une rue dans le quartier très populaire de Lille-Sud, ce qui convient bien au personnage qui s'engagea, sa vie durant, pour améliorer le sort des populations besogneuses. En revanche, aucune rue ne porte le nom de Dweira Bernson (1871-1944), son épouse et également médecin, ni celui de sa fille Reysa (1904-1944), physicienne et astronome reconnue dans les années 1930. Pourtant, toutes deux ont consacré leur vie, comme leur mari et père, à l'amélioration du sort des « classes laborieuses » pour que celles-ci puissent accéder à la santé et à la connaissance scientifique. Elles n'ont guère occupé de postes politiques régionaux, à la différence de Désiré Verhaeghe, mais elles n'ont pas moins agi que lui, dans l'ombre pour ensuite tomber dans l'oubli. La seule trace plus ou moins visible qu'elles laissent est une mention dans Le Mémorial de la Déportation des Juifs de France ${ }^{1}$, à la liste du convoi 69 qui les emmène, le 7 mars 1944, à Auschwitz ainsi que

\footnotetext{
* Université de Lille, Sciences humaines et sociales. Je dois remercier, pour leur aide serviable et efficace, Isabelle Kosiak aux archives municipales de Lille, Hervé Passot et Ophélie Gérard aux archives départementales du Nord.

Société Astronomique de France. Fondateur du Planétarium de Montpellier et de la revue Planétariums.

${ }^{1}$ Édité par Beate et Serge Klarsfeld, 1978, $1^{\text {ère }}$ édition.
} 
leur nom gravé sur le mur des déportés du Mémorial de la Shoah à Paris. Dweira (ou Déborah) était juive, épouse d'《aryen», et Reysa était « demi-juive », pour copier le vocabulaire nazi, mais cela ne leur fut d'aucun secours: la mère médecin, dévouée envers les populations déshéritées, et la fille astrophysicienne, au service de l'instruction des enfants, ne méritaient que la mort selon l'idéologie raciste nazie.

\section{Désiré Verhaeghe, homme politique lillois et médecin hygiéniste}

Désiré Verhaeghe était un homme du Nord, né à Estaires, commune située non loin d'Hazebrouck, en Flandre française où le catholicisme était prégnant ${ }^{2}$, d'où son père était originaire. Celui-ci était employé des contributions indirectes selon l'acte de naissance de Désiré, né le 17 mai $1874^{3}$. La famille n'était donc pas très fortunée mais suffisamment pour payer des études de médecine à son fils.

Le sujet de la thèse de médecine de Désiré Verhaeghe évoque déjà l'intérêt que portait l'étudiant en médecine sur le sort des populations ouvrières: De l'alcoolisation, effets, causes, remèdes (étude de pathologie sociale) ${ }^{4}$. Elle fut soutenue le 24 juillet 1899 , à la faculté de médecine et de pharmacie de Lille. Toutefois, cette soutenance semble s'être heurtée à une certaine réticence de la part du jury. Dans une lettre du 11 juillet 1899, le doyen de la faculté de médecine et de pharmacie de Lille écrivait au recteur de l'académie de Lille : «J'ai l'honneur de vous adresser d'urgence le manuscrit de la thèse de $M$. Verhaeghe, qui ne présente plus, comme travail inaugural, qu'une petite partie purement scientifique et médicale » et en conséquence il demandait au recteur de donner son «approbation pour l'impression » ${ }^{5}$. On peut soupçonner, de la part du jeune thésard, un débordement de son sujet en introduisant, éventuellement, des remarques politiques socialistes puisque son travail fut d'abord « refusé par la censure décanale et rectorale $»^{6}$. Toutefois la

\footnotetext{
2 L'abbé Lemire, éminent catholique social, fut député de la circonscription d'Hazebrouck de 1893 à 1928 et maire de la ville de 1914 à 1928, il était lui-même originaire de Vieux-Berquin, non loin d'Estaires.

${ }^{3}$ État civil de la municipalité d'Estaires.

${ }^{4}$ Archives municipales de Lille (désormais AML), 1 D 4.

${ }^{5}$ Archives départementales du Nord (désormais AD 59), 2 T 1092.

${ }^{6}$ Mention figurant à la suite du titre de sa thèse sur le catalogue de la Bibliothèque nationale de France : «Travail présenté comme thèse de doctorat devant la Faculté de médecine de Lille et refusé par la censure décanale et rectorale, imprimé en 1900, 279 p. ».
} 
soutenance eut bien lieu deux semaines après la levée de censure par le doyen de la faculté.

Cet incident ne peut étonner car, très tôt, l'étudiant en médecine s'engagea dans un parcours politique, chez les socialistes. Dès 1895, il se fit remarquer "pour ses idées très avancées et ses tendances internationalistes » précise un rapport de police $^{7}$. Ce qui expliquerait sa tentative de réforme militaire pour insuffisance visuelle ${ }^{8}$, tentative restée vaine puisqu'en 1895-1896 il était incorporé dans l'infanterie comme soldat de $2^{\mathrm{e}}$ classe $^{9}$. En cette année 1895 , il « est affilié au groupe des étudiants socialistes collectivistes » et, le 25 avril 1895, il participa à l'assemblée générale de l'Union des Étudiants de l'État qui se tint à Lille. Il y manifesta clairement ses tendances internationalistes en interpellant le bureau qui n'avait pas invité les étudiants allemands aux fêtes universitaires des $1^{\text {er }}$ et 2 juin $^{10}$. Épouser une étrangère ne devait donc susciter aucun état d'âme chez cet étudiant. En 1899, il était président du groupe des étudiants socialistes de Lille ${ }^{11}$. Il tenta une carrière politique en se présentant, vainement, aux élections législatives de 1902 mais, avant guerre, son rôle politique resta limité ${ }^{12}$. Ce ne fut qu'au retour de la guerre qu'il se lança pleinement dans la vie politique lilloise et ceci pendant une dizaine d'années, jusqu'à son décès.

En effet, à son décès, Le Réveil du Nord, quotidien socialiste, cite toutes ses fonctions : « En novembre 1919, M. Désiré Verhaeghe était élu conseiller général du Canton de Lille-Sud ; en décembre 1919, conseiller municipal de Lille, puis quelques semaines après, adjoint à l'Hygiène et à la Solidarité Sociale ${ }^{13}$. Il représentait la SFIO à laquelle il adhérait. Son acte de décès ajoute qu'il était chevalier de la Légion d'Honneur et décoré de la Croix de Guerre ${ }^{14}$. Si dans sa jeunesse il tenta d'éviter l'incorporation militaire, dès lors que son pays était en danger ses

\footnotetext{
${ }^{7}$ Rapport de police sur le candidat aux législatives de 1902. AD 59, M 154 / 78 c.

${ }^{8}$ Ibid, même rapport.

${ }^{9} \mathrm{AD} 59,1 \mathrm{R} / 02430$, son carnet militaire rendant compte de ses services à l'armée et notamment pendant la Première Guerre mondiale.

${ }^{10}$ Rapport de police sur Désiré Verhaeghe du 7 novembre 1895. AD 59, M 154 / 78 c. Il ne fut d'ailleurs pas suivi par le bureau qui rejeta sa proposition. Dans le contexte d'une revanche sur la défaite de 1870 et d'une affaire Dreyfus naissante, l'amitié francoallemande n'était pas encore prisée même chez les socialistes.

${ }^{11}$ AML, 1 D 4, fiche de l'OURS (Office Universitaire de Recherche Socialiste) concernant Désiré Verhaeghe.

${ }^{12}$ Rapport de police du 24 avril 1902. AD 59, M 154 / 78 c. « M. Verhaeghe passe pour un ambitieux » mais il ne prit pas la parole pour ménager sa clientèle, selon ce rapport.

${ }^{13}$ Le Réveil du Nord, 16 avril 1927. Désiré Verhaeghe est décédé le 14 avril 1927.

${ }^{14}$ Son dossier aux AML, 1 D 4, contient en outre six autres médailles.
} 
sentiments patriotiques semblent évidents, lorsqu'on a connaissance de ses états de service dans l'armée durant la guerre. Le 10 janvier 1919, il fut cité à l'ordre des Formations sanitaires du $18^{\mathrm{e}}$ Corps d'armée pour avoir assumé les fonctions de médecin "chef d'une ambulance de l'avant » durant les deux années 1917-1918 ${ }^{15}$. « Médecin d'une très belle tenue morale et spécialiste d'une haute valeur professionnelle [... a constamment donné à son personnel l'exemple d'un beau dévouement allié à une parfaite insouciance du danger ${ }^{16}$. Il avait alors 43-44 ans. Et le 4 septembre 1920, il était inscrit aux tableaux spéciaux de la Légion d'Honneur, au titre de médecin major de $2^{\mathrm{e}}$ classe, $1^{\text {er }}$ Corps d'armée ${ }^{17}$.

Le bon soldat Verhaeghe fut également un excellent médecin. Déjà l'étudiant s'était fait remarquer par un parcours brillant: « [...] il a brillamment passé tous ses examens. Il avait d'ailleurs la réputation d'être un des meilleurs étudiants de son époque ${ }^{18}$. Le rapport de police, établi à l'occasion des élections législatives de 1902, informe qu'il jouissait d'une grande considération dans «les quartiers populaires de Wazemmes et des Postes » auprès d'une «clientèle moins riche que nombreuse ${ }^{19}$.

Le Réveil du Nord du 15 avril 1927 rappelle que «Le docteur Verhaeghe s'était dévoué corps et âme depuis plus de trente ans ${ }^{20}$ pour soulager les misères des humbles et des déshérités ». Il œuvra aussi huit années à la mairie de Lille comme adjoint «à la solidarité sociale, à l'assistance et à l'hygiène » ajoute le journal à la même date ${ }^{21}$. Deux de ses fondations sont à retenir. D'abord le secrétariat ouvrier d'hygiène, sis

\footnotetext{
${ }^{15}$ Ordre de la Direction du Service de Santé, daté du 10 janvier 1919. AML, 1 D 4. Selon sa fiche matriculaire et d'affectation (AD 59, 1 R / 02430), il fut nommé médecin aide major de $2^{\mathrm{e}}$ classe de réserve par décret du 3 février 1900, promu médecin aide major de $1^{\text {ère }}$ classe de réserve le 6 août 1904 et rappelé à activité par décret du $1^{\text {er }}$ août 1914, au service de santé de Lille puis affecté à Reims le 19 août 1914 et replié sur Le Mans le 2 septembre 1914, puis affecté à une ambulance de la $\mathrm{V}^{\mathrm{e}}$ armée, en 1916.

${ }^{16}$ Ordre de la Direction du Service de Santé, daté du 10 janvier 1919. Il s'était distingué à : « Glennes 1917, Boyenville avril 1918, Royallieu juin 1918 ». AML, 1 D 4.

${ }^{17}$ Extrait du Journal officiel du 4 septembre 1920. AML, 1 D 4.

${ }^{18}$ Rapport de police du 24 avril 1902. AD 59, M 154 / 78c. Il fut effectivement classé premier au concours d'externat du 5 décembre 1898: AD 59, 96 J 518 / 2, nomination des internes et des externes.

${ }^{19} \mathrm{AD} 59, \mathrm{M} 154$ / 78c. Le village agreste de Wazemmes fut rattaché à la commune de Lille lors de l'haussmannisation de la ville et devint un quartier industriel où proliféra un habitat de courées insalubres, peuplées d'ouvriers très pauvres dont un grand nombre étaient Belges.

${ }^{20}$ En 1927, il était docteur en médecine depuis 28 ans.

${ }^{21}$ Voir aussi le cahier des délibérations du Conseil municipal de Lille : il est adjoint à l'hygiène puis premier adjoint en 1925. AML, 1 D 2 / 124.
} 
place Vanhoenacker, en plein quartier ouvrier de Moulins-Lille où une clinique recevait des «malheureux malades, accidentés du travail, mutilés de guerre », et fournissait des soins gratuits ${ }^{22}$. Ensuite l'école de plein air qui porta son nom et qu'il ne vit pas fonctionner. En effet, la première pierre fut posée en février 1929, deux ans après sa mort, et l'école fut inaugurée le 27 septembre 1931 ; elle n'ouvrit que le $1^{\text {er }}$ décembre 1931, dans un quartier sud de la ville toujours proche des habitations ouvrières ${ }^{23}$. Au terme de sa carrière de médecin, Désiré Verhaeghe avait respecté sa promesse exprimée dans la dédicace de sa thèse : «À mes Frères du Prolétariat manuel qui n'ont pas eu le bonheur de pouvoir s'approcher de la source féconde de Vérité et de Beauté ; à qui je suis redevable des loisirs de ma jeunesse privilégiée ; devant qui je me déclare responsable de l'emploi que j'en ai fait, de l'usage que je ferai dans l'avenir du savoir acquis $»^{24}$.

Son action auprès des populations ouvrières et déshéritées de Lille s'inscrivait dans la politique de la médecine hygiéniste qui se développait à la fin du $\mathrm{XIX}^{\mathrm{e}}$ siècle et surtout dans les premières décennies du $\mathrm{XX}^{\mathrm{e}}$ siècle. Elle fut à l'origine des écoles de plein air où l'on accueillait les enfants pauvres à la santé déficiente, ainsi qu'à l'origine des colonies de vacances qui hébergeaient les écoliers des villes depuis que les lois de Jules Ferry leur imposaient l'école et en conséquence des vacances estivales. À l'école de plein air Désiré-Verhaeghe, qui fonctionna durant tout le $\mathrm{XX}^{\mathrm{e}}$ siècle, le personnel veillait aux progrès scolaires des enfants mais aussi à leur santé, notamment leur alimentation, et à leur développement physique ${ }^{25}$.

\section{Dweira Bernson, épouse Verhaeghe, médecin des ouvrières}

Dans son engagement, au moins pour sa partie médicale, le docteur Désiré Verhaeghe n'était pas seul. Son épouse Dweira née Bernson suivait le même parcours.

Et pourtant, elle semble n'exister que par la présence de son mari. Nous l'avons vu, la ville de Lille ne conserve pas son souvenir. Bien que proche du socialisme, le Maitron ne lui consacre aucune notice sinon une

\footnotetext{
${ }^{22}$ Le Réveil du Nord du 15 avril 1927.

${ }^{23}$ AML, dossiers sur l'école de plein air : 4 M 8/145 et 4 M 8/152.

${ }^{24}$ AML, 1 D 4.

${ }^{25}$ Sur l'école de plein air Désiré Verhaeghe, voir Nadège Loiselle, L'école de plein air Désiré Verhaeghe de Lille, Mémoire d'élèves de l'école d'architecture de Versailles, sous la direction d'Anne-Marie Châtelet, 1999-2000, avec un vol. d'annexes.
} 
vague mention dans celle que le dictionnaire a publiée sur son mari ${ }^{26}$. Le dossier sur Désiré Verhaeghe constitué aux archives municipales de Lille ne contient aucun renseignement sur l'épouse de l'adjoint au maire de Lille. Les comptes rendus du décès du médecin et édile de la ville n'évoquent pas la présence de Dweira Bernson, ni même celle de sa fille. Toutefois, cette dernière assista à la pose de la première pierre de l'école de plein air qui portera le nom de son père comme le rapporta la presse ; mais rien sur sa femme Dweira ${ }^{27}$. Était-elle dans l'assistance?

Dweira (ou Déborah forme francisée de son prénom) Bernson semble bien seule et isolée, à Lille, en cette fin du XIX ${ }^{\mathrm{e}}$ siècle. Son nom apparaît pour la première fois dans les archives grâce à son inscription en faculté de médecine. Aux archives départementales du Nord sommeille un arrêté du recteur de l'académie de Lille, daté du 7 janvier 1898 ; il informe que: « $\mathrm{M}^{\text {lle }}$ Bernson Déborah, étudiante en médecine, est déléguée dans les fonctions de moniteur d'anatomie à la faculté de médecine de l'Université de Lille », son service devant cesser le 31 octobre 1898. Il lui a permis de toucher une indemnité annuelle de 200 francs ${ }^{28}$. D'autre part, aux mêmes archives se trouve une liste d'étudiants inscrits en médecine pour l'année scolaire 1898-1899. Sur 35 noms, on peut lire celui de deux jeunes filles dont « $\mathrm{M}^{\text {elle }}$ Bernson (russe) », l'autre étant « française » précise la liste ${ }^{29}$. Enfin, le 20 mai 1899 à la faculté de médecine et de pharmacie de Lille, Deborah Bernson soutint sa thèse de médecine ; la couverture de ce document indique qu'elle est née le 3 janvier 1871 à Brest-Litewki (Russie) ${ }^{30}$, en fait Brest-Litovsk en Biélorussie. Ainsi donc, bien qu'aucune source ne nous renseigne sur son arrivée en France, il nous est permis de supposer qu'elle y arriva, déjà adulte, à la fin de la décennie 1890 .

Nulle part nous ne trouvons un indice sur la présence de ses parents ou de sa famille dans le Nord ou en France. La communauté juive de

\footnotetext{
${ }^{26}$ Jean Maitron, Dictionnaire biographique du mouvement ouvrier français, vol. 15, 1871-1914, Paris, éd. de l'Atelier.

${ }^{27}$ Le Réveil du Nord du 18 février 1929 : le maire Roger Salengro « rend un délicat hommage à la mémoire du docteur Verhaeghe ainsi qu'à sa fille qui assistait à la cérémonie ». AML, 4 M 8/145.

${ }^{28}$ AD 59, $2894 \mathrm{~W} 53$, notice individuelle de Bernson Divora (sic).

${ }^{29}$ AD 59, $2894 \mathrm{~W} 7$ / 2, liste des étudiants inscrits en faculté de médecine pour l'année scolaire 1898-1899.

${ }^{30}$ AML, 1 D 4, dossier Désiré Verhaeghe. Ce dossier ne contient que la couverture de la thèse dont un exemplaire se trouve à la bibliothèque universitaire de Lille 2 , mais il n'a pas été possible de consulter la thèse.
} 
Lille semble même ignorer sa présence ${ }^{31}$. S'est-elle manifestée auprès de ladite communauté ? Pourtant, elle a rencontré des hommes qui en faisaient partie : le docteur Wertheimer, président de la communauté juive de Lille de 1885 à 1889, ainsi que Bernard Wellhoff qui figura sur la liste des électeurs du consistoire de Lille pour l'année $1905^{32}$. Le premier était médecin et faisait partie du jury de thèse de son mari en 1899, le second était receveur municipal de Lille sous le mandat du socialiste Gustave Delory (1896-1904 et 1919-1925), il fut le témoin qui assista Désiré Verhaeghe lors de la déclaration de naissance de sa fille Reysa $^{33}$. Une fois encore, c'est par l'intermédiaire de son mari que Dweira Bernson rencontra des personnalités de la communauté juive de Lille : le docteur Wertheimer pour des raisons professionnelles et Bernard Wellhoff pour son militantisme socialiste et aussi maçonnique puisque Désiré Verhaeghe et lui appartenaient tous deux à une loge ${ }^{34}$.

Dweira Bernson était donc originaire de Brest-Litovsk, en Biélorussie mais l'adjonction de Litovsk illustre l'attachement de la ville, et de la communauté juive, à la Lituanie à laquelle la Biélorussie fut, un temps, rattachée. Lors du partage de la Pologne, en 1795, la Lituanie et la Biélorussie furent dévolues à la Russie qui mena, durant tout le $\mathrm{XIX}^{\mathrm{e}}$ siècle, une politique de russification. Dès la fin du Moyen Âge, la présence de communautés juives prit de l'importance et durant les XVII et $\mathrm{XVIII}^{\mathrm{e}}$ siècles, à l'instar de la capitale de la Lituanie Vilna, BrestLitovsk devint un centre religieux juif de grande envergure. Ce fut dans ces deux villes que se développa le courant des mitnagdim opposés au hassidisme qui prenait naissance en Pologne et dans les régions plus méridionales $^{35}$. En 1889, plus de la moitié de la population de Brest-

\footnotetext{
${ }^{31}$ Lors de mes recherches pour ma thèse d'État, je n'ai trouvé aucun indice la concernant dans les archives consistoriales, nationales, départementales et municipales (sauf le dossier AML, 1 D 4 sur Désiré Verhaeghe). Danielle Delmaire, Les communautés juives septentrionales, 1791-1939, naissance, croissance épanouissement, thèse soutenue en janvier 1998, à l'université Charles-de-Gaulle - Lille 3.

${ }^{32} \mathrm{AD} 59,7 \mathrm{~V} 50$.

${ }^{33}$ AML, 7 E 543, acte de naissance de Reysa Verhaeghe.

${ }^{34}$ Le Réveil du Nord du 16 avril 1927 pour Désiré Verhaeghe. Sur Bernard Wellhoff voir Danielle Delmaire, Les communautés juives de la France septentrionales au XIX siècle (1791-1914). L'entrée dans la Nation, Paris, L'Harmattan, 2008, pp. 237-238.

${ }^{35}$ Le hassidisme préconisait des pratiques religieuses plus strictes et extériorisées que celles plus académiques des écoles juives ou yéshivot. Les mitnagdim (opposants en hébreu), craignant des déviances religieuses, s'opposèrent parfois durement aux hassidim durant une bonne partie du $\mathrm{XIX}^{\mathrm{e}}$ siècle. Le Gaon de Vilna est une grande figure du courant des mitnagdim.
} 
Litovsk était juive ${ }^{36}$. À partir de la décennie 1880, suite à l'assassinat du tsar Alexandre II en 1881, des pogroms déstabilisèrent les communautés juives et les étudiants juifs furent soumis à un numerus clausus qui leur fermait les portes des universités. Aussi un grand nombre d'entre eux émigrèrent-ils vers les pays occidentaux, notamment en France ; les filles étaient encore plus nombreuses car plus discriminées que les garçons lorsqu'il s'agissait d'études supérieures ${ }^{37}$. On peut donc supposer que la jeune Dweira Bernson suivit ce parcours migratoire afin de poursuivre des études de médecine en France. La date de son arrivée à Lille et le choix de cette ville nous restent inconnus ${ }^{38}$. A-t-elle débuté ses études de médecine en Biélorussie ou est-elle arrivée en France pour les commencer? En 1898, lorsque son nom apparaît sur les listes de la faculté de médecine de Lille, elle est déjà «moniteur d'anatomie » à l'âge de 27 ans.

Le sujet de sa thèse en dit long sur l'engagement social de la future docteure en médecine: Nécessité d'une loi protectrice pour la femme ouvrière avant et après ses couches (Étude d'hygiène sociale) ${ }^{39}$, le jury était présidé par le professeur Debierre, celui-là même qui présidait le jury de thèse de Désiré Verhaeghe. Comme son compagnon, Dweira Bernson consacra sa carrière de médecin à l'amélioration sanitaire et hygiénique des populations ouvrières défavorisées et plus particulièrement des femmes soumises à de plus dures conditions de travail que les hommes pour un salaire souvent inférieur. On peut deviner une certaine complicité entre les deux étudiants en médecine, au moins une certaine admiration de la part de Désiré Verhaeghe qui dédiait sa thèse «À mon amie le docteur Déborah Bernson » en même temps qu'à ses « Frères du Prolétariat manuel ». Son amie venait tout juste d'obtenir le titre de docteur quand lui présentait sa thèse à un jury réticent. Il faut reconnaître à Désiré Verhaeghe un certain courage pour avoir comme amie une juive étrangère, en pleins remous de l'affaire Dreyfus qui

\footnotetext{
${ }^{36}$ Jewish virtual library, http///www.jewishvituallibrary.org/jsource/judaica/ejud.html. Site consulté le 23 août 2016.

${ }^{37}$ Lire à ce sujet Catherine Goussef, «Les Juifs russes en France. Profil et évolution d'une collectivité », Archives Juives, 2001/2, vol. 34, pp. 4-16, et surtout Irina et Dimitri Gouzevitch, «Étudiants, savants et ingénieurs juifs originaires de l'Empire russe en France (1860-1940) », Archives juives, 2002/1, vol. 35, pp. 120-127.

${ }^{38}$ Pour ma thèse d'État, op. cit., j'avais mis en évidence un courant migratoire de juifs originaires de Russie, vers Lille, dans les années 1890 et 1900. Il me fut difficile de déterminer si elle en faisait partie.

${ }^{39}$ Selon le catalogue de la Bibliothèque nationale de France, elle fut rapidement éditée dès 1899 .
} 
agitaient les rues de Lille. Le procès de Rennes qui condamna Dreyfus avec des circonstances atténuantes se termina le 9 septembre 1899. C'est dire que les mois de leur soutenance de thèse furent agités par l'Affaire. Certes, globalement, l'université d'État où ils étudiaient manifestait sa sympathie pour le capitaine, et les antidreyfusards se recrutaient essentiellement dans les rangs de l'Université catholique, mais les guesdistes lillois ne s'intéressaient pas particulièrement au sort d'un militaire, de surcroît de famille bourgeoise ${ }^{40}$.

Peu de temps après, le 11 avril 1900, Désiré et Dweira se marièrent à Ixelles dans la banlieue de Bruxelles. Désiré Verhaeghe était alors domicilié à Lille et ses parents étaient consentants. Dweira Bernson résidait à Ixelles depuis six mois, c'est-à-dire peu de temps après sa soutenance de thèse à Lille. Le consentement de ses parents n'était pas requis, probablement à cause de l'éloignement et de la difficile communication avec des personnes définies comme «sujets russes", selon l'acte de mariage. Leurs quatre témoins étaient domiciliés à Ixelles. L'un d'eux peut retenir notre attention, Nicolas Michoff, étudiant, à cause de la consonance russe de son patronyme sans que l'on puisse en tirer un quelconque renseignement sur le cercle d'amis des époux ${ }^{41}$. Le 28 septembre 1904, Désiré Verhaeghe présenta en mairie de Lille « un enfant du sexe féminin, né le vingt six septembre [...] de lui déclarant et de Dweira Bernson son épouse [...] tous deux domiciliés à Lille rue Solférino 249. Auquel enfant il a déclaré donner le prénom de Reysa $»^{42}$. Les jeunes parents donnèrent à leur fille le prénom de la mère de Dweira, tel qu'il apparaissait sur leur acte de mariage. C'est le seul témoignage de l'attachement de la jeune immigrée juive, originaire de Russie, à sa famille.

Dans les années 1920, le couple s'est disloqué. D’après les documents établissant la succession de Désiré Verhaeghe, ce dernier avait accordé, en cas de décès, une assurance à Paul Janny né le 25 juin $1921^{43}$. Sur son acte de naissance, l'enfant est déclaré «nat» ou naturel

\footnotetext{
${ }^{40}$ Sur l'antisémitisme des ouvriers socialistes lillois, condamné par Guesde lui-même, lire Danielle Delmaire, Antisémitisme et catholiques dans le Nord pendant l'affaire Dreyfus, Lille, Presses Universitaires de Lille, 1991, notamment les pp. 182-187.

${ }^{41}$ Acte de mariage de l'état civil de la commune d'Ixelles, $\mathrm{RC} \mathrm{n}^{\circ}$ 959. L'utilisation de l'onomastique reste toujours très délicate. Les époux passèrent une convention matrimoniale chez un notaire à Saint Josse Ten Noode, le 6 avril 1900.

${ }^{42}$ Acte de naissance de Reysa Bernson, AML, 7 E 543.

${ }^{43}$ Voir le dossier de la succession de Désiré Verhaeghe aux AD 59, 3 Q 318 / 393 et 394, 3 Q 318 / 548.
} 
mais c'est le docteur Désiré Verhaeghe qui fait la déclaration car «ayant assisté à l'accouchement ${ }^{44}$. La consultation des annuaires RavetAnceau $^{45}$ confirme la séparation puisque les deux époux n'avaient plus la même adresse dans les années 1920 et ce fut au domicile de Geneviève Janny que décéda Désiré Verhaeghe. Toutefois, dans aucun document il n'est fait mention d'un divorce: ni sur l'acte de décès de Désiré Verhaeghe, ni dans le dossier de sa succession, ni même dans les mentions marginales de son acte de naissance ${ }^{46}$. Partout il est désigné comme l'époux de Dweira Bernson.

Il n'empêche qu'ensemble, dans les années d'avant-guerre ${ }^{47}$, ou séparément, après la guerre, les époux ont travaillé pour améliorer le sort de la population ouvrière de Lille. Selon l'annuaire Ravet-Anceau, la docteure Bernson, en association avec le docteur Doumer, dirigeait, en 1914, « la clinique pour les maladies des femmes » sise 28 rue SaintSauveur ainsi que la Ligue de la Protection de l'Enfance qui jouxtait la clinique au $n^{\circ} 26$. La docteure en médecine s'était donc spécialisée en gynécologie et en pédiatrie, finalement dans la continuité de sa thèse. Désiré Verhaeghe avait, pour sa part, ouvert un «secrétariat ouvrier d'Hygiène de Lille », 1 place Vanhoenacker pour soigner les accidentés $\mathrm{du}$ travail et les mutilés de guerre ${ }^{48}$ et il s'était spécialisé dans les maladies des yeux, thème éloigné de son sujet de thèse. La rue SaintSauveur et la place Vanhoenacker étaient situées dans deux quartiers très populaires de Lille. De nombreuses courées insalubres et misérables débouchaient sur la rue Saint-Sauveur et autour de la place Vanhoenacker étaient rassemblées les pauvres habitations d'ouvriers des industries textiles. Chacun dans son domaine soignait et soulageait les malades pauvres, dans un contexte de mortalité infantile encore élevée. L'infrastructure hospitalière pour les accouchements restait très insuffisante ou était entre les mains d'œuvres catholiques charitables ${ }^{49}$,

\footnotetext{
${ }^{44}$ Acte de naissance de Paul Janny, AML, 7 E 700. Le 29 août 1921, sa mère Geneviève Janny « a déclaré [le] reconnaître pour son fills », acte d'état civil, AML, 7 E 702.

${ }^{45}$ Ces annuaires qui couvrent tout le département du Nord publient, depuis les années 1850 , les adresses des habitants par commune, selon les rues et selon la profession.

${ }^{46}$ Respectivement : AML, 1 D 4 ; AD 59, 3 Q 718 / 1012 où Dweira Bernson est notée son épouse survivante ; état civil de la municipalité d'Estaires. Et en 1931, l'annuaire Ravet-Anceau la nommait $\mathrm{M}^{\mathrm{me}}$ Veuve Verhaeghe Bernson.

${ }^{47}$ Dans ces années-là, les annuaires Ravet-Anceau les localisent toujours au même domicile.

${ }^{48}$ Le Réveil du Nord, 16 avril 1927.

${ }^{49}$ Malgré son ancienneté, l'ouvrage de Pierre Pierrard, Lille et les Lillois, Paris, Bloud et Gay, 1967, fournit encore de précieux renseignements sur ce sujet, notamment les
} 
voire protestantes à Fives, autre quartier ouvrier également très populeux $^{50}$.

Selon l'annuaire Ravet-Anceau, la docteure Bernson était encore présente en 1931, après le décès de son mari, à la clinique de la place Vanhoenacker, mais elle habitait avec sa fille Reysa Bernson (et non Verhaeghe), étudiante, au 219 boulevard de la Liberté. Cependant, le recensement de la même année, plus fiable, les localise au 217 du Bd de la Liberté ${ }^{51}$. Dans les années trente, elle poursuivit son combat pour adoucir le sort des femmes et des enfants. La Bibliothèque nationale de France conserve une trace de la continuité de ses activités en faveur de l'enfance malheureuse. Dans la revue Le mouvement sanitaire, de juin 1938, elle plaida «pour la sauvegarde de la vie de l'enfance sans défense » et elle y rappelait qu'en mars 1936 elle avait déjà signalé dans « une lettre ouverte adressée à un grand nombre de journaux le danger que représente la publication détaillée [...] des meurtres d'enfants par leurs proches » car cette publicité pouvait inciter d'autres femmes à vouloir supprimer leur bébé non voulu. En cette occasion, elle réclamait, comme elle le faisait dans sa thèse, l'intervention des législateurs : elle souhaitait « qu'une loi soit élaborée afin que la publication des crimes se limite à une simple information dont l'emplacement, le titre, la teneur n'attirent en rien l'attention du lecteur $»{ }^{52}$.

En revanche, rien ne transparaît sur ses positions politiques que l'on peut tout de même supposer à gauche et socialistes. Mais à l'époque où, en France, le droit de vote féminin n'était pas encore reconnu, les femmes n'exerçaient que très rarement une responsabilité politique. D'où leur effacement en général de la scène politique et celui de Dweira

pp. 242-245. «En 1900, Lille arrivait en tête des grandes villes de France pour la mortalité infantile ». D'autre part, suite à une épidémie meurtrière de diphtérie à Lille, un Institut Pasteur fut inauguré, à Lille, en 1899 avec pour directeur le docteur Calmette, élève de Pasteur (site électronique de l'Institut Pasteur, consulté le 6 mars 2017).

${ }^{50}$ «Il meurt à Lille 28\% des enfants n'ayant pas atteint l'âge de un an. En 1902, il y a eu à Lille 5906 naissances, 485 mort-nés, notez ce chiffre énorme ; 1/12 des enfants ne naissent pas viables », selon le témoignage du pasteur Nick, desservant la paroisse protestante de Fives, cité dans Henri Roser, Monsieur Nick, cinquante-sept ans d'une vie dans le faubourg de Fives-Lille, 1897-1954, Paris, éditions de la Mission Populaire Évangélique de France, 1982, p. 19. Le pasteur Nick appartenait au courant protestant $\mathrm{du}$ christianisme social, très proche des thèses socialistes pour ce qui concerne l'organisation de la société.

${ }^{51}$ AML, recensement de 1931, 1 F 29.

${ }^{52}$ L'Hygiène par l'exemple, 1938, pp. 184-185, rend compte de son intervention dans Le mouvement sanitaire. 
Bernson en particulier, rendue peu visible par l'aura de son mari. Mais on peut se demander quels furent ses sentiments, à partir de 1917, envers son pays d'origine qui vivait sa Révolution prolétarienne?

L'on peut néanmoins constater un positionnement féministe, par sa volonté d'entreprendre de longues études coûteuses mais qui lui apporteront une certaine indépendance, par son engagement auprès des femmes en couches et des enfants. Ceci expliquerait qu'elle conserva très longtemps son patronyme: elle est toujours dénommée et recensée « docteur Bernson ». Ce n'est qu'après le décès de son mari qu'elle apparaît sous l'appellation de «veuve Verhaeghe » dans Ravet-Anceau. Dans le recensement de 1931, elle figure au nom de Verhaeghe avec la nationalité russe, tandis que Reysa est «étudiante» et « française » 53 . Plus étonnant, sans doute, est l'identité de sa fille Reysa qui pourtant fut reconnue par son père mais qui constamment est dénommée Bernson.

\section{Reysa Bernson, physicienne et astronome}

Reysa Bernson était licenciée ès sciences, férue d'astronomie. Lors d'un voyage à Paris en 1922, elle assista à une conférence de Camille Flammarion $^{54}$ et échangea quelques mots avec lui. Cette rencontre déterminante à l'âge de 18 ans fut à l'origine de sa vocation. Un an plus tard, elle fonda l'Association Astronomique du Nord (AAN) à Lille. D'une énergie débordante, avec les concours de Gabriel Delmotte, Henri d'Halluin et Émile Delahaye, elle s'occupa du transfert des instruments de l'Observatoire de Robert Jonckheere, de Hem à Lille, pour aménager l'Observatoire de l'AAN ${ }^{55}$.

Lauréate du prix de la Société Astronomique de France (dont le prix Henri Rey, 1932) ${ }^{56}$, passionnée par les étoiles variables, elle publia

\footnotetext{
${ }^{53}$ AML, recensement de 1931, 1 F 29.

${ }^{54}$ Camille Flammarion (1842-1925) est un célèbre astronome français qui vulgarisa de nombreuses découvertes scientifiques. Voir Jean-Michel Faidit, Ces Français dans la Lune... Cratères et Biographies, Toulon, Les Presses du Midi, 2013, p. 92 concernant Camille Flammarion. À propos de Reysa Bernson, Idem, « Reysa Bernson et son œuvre fondatrice pour les Planétariums en France », L'Astronomie, vol. 129, avril 2015, 82, pp. 40-45.

${ }^{55}$ L'annuaire Ravet-Anceau de 1930 présente pour la première fois l'AAN, fondée en 1923. Dès sa fondation, « Melle R. Bernson, étudiante et licenciée ès sciences » en était secrétaire. Elle n'avait que 19 ans et c'était immédiatement après sa rencontre avec $\mathrm{C}$. Flammarion. L'AAN était localisée, en 1930, 50 rue Gauthier de Châtillon, à 1'Institut de Physique de la faculté des sciences, non loin du boulevard de la Liberté où Reysa habitait avec sa mère.

${ }^{56}$ L'Astronomie, 1932, p. 342.
} 
de brefs comptes rendus d'observations (éclipses de soleil et de lune, novae, comètes, pluie d'étoiles filantes de 1933) ainsi que de ses voyages astronomiques, notamment en Allemagne et en Pologne en $1932^{57}$. Cependant, Reysa écrivit peu. Femme de terrain, elle préféra agir. Son principal article, «L'astronomie et la jeunesse », est inséré en deux parties, dans les bulletins d'août et de septembre 1937 de L'Astronomie, quatorze pages en tout mais d'une richesse et d'une densité inouïes. En septembre 1938 parut un second article qui complétait le premier : «Une réalisation d'astro-scoutisme. Le Groupe Camille Flammarion des Éclaireurs de France et son Planétarium ${ }^{58}$.

Au-delà des mots, à travers les lignes, on ressent un souffle, une vision novatrice pour diffuser l'astronomie auprès des jeunes avec le développement des planétariums. Après l'invention, en 1923, du planétarium de Bauersfeld, ingénieur allemand (1879-1959), ces nouveaux théâtres d'étoiles se répandirent, d'abord en Allemagne puis dans les grandes capitales. Une vingtaine de grands équipements furent réalisés entre 1923 et 1939. En France, il fallut attendre 1937 pour voir s'installer un planétarium à l'Exposition universelle, confié à Reysa. Dès lors, les planétariums se multiplièrent, les élèves constituant les trois quarts de leur public. Encore fallait-il que l'astronomie fût introduite dans les programmes scolaires.

Sur ces deux tableaux, cette femme extraordinaire joua un rôle décisif. Elle fut un tourbillon d'énergie. Elle visita une centaine d'écoles bénévolement dans le quart nord-est de la France entre 1929 et 1939. Près de 20000 élèves furent initiés au ciel! Dans ces années trente, c'était nouveau. Et à cette échelle, c'était unique. À l'image de l'investissement politique de ses parents, Reysa interpella le ministre de l'Éducation nationale, Jean Zay, qui fit acquérir plusieurs de ses instruments pédagogiques pour introduire l'astronomie à l'école ${ }^{59}$. Visionnaire, elle imagina aussi le développement des planétariums itinérants et l'astronomie en tant que loisir pour la jeunesse.

Son activité en faveur des élèves démarra en 1929 avec le concours de l'AAN, alors qu'elle était en villégiature dans le Boulonnais ${ }^{60}$. Son but? Chercher à sensibiliser les jeunes là où ils étaient : établissements

\footnotetext{
${ }^{57}$ Revue de l'AAN pour cette année.

${ }^{58}$ L'Astronomie, 1938, pp. 411-414.

${ }^{59}$ Ibid., p. 412.

${ }^{60}$ Reysa Bernson, «L'astronomie et la jeunesse », L'Astronomie, 1937, pp. 362-370 et pp. 424-428, ici p. 369.
} 
d'enseignement et mouvements de jeunesse avec des conférences, des observations et des films. Elle élabora une conférence vivante: « Excursion au pays des astres » agrémentée de projections et d'appareils de démonstration qu'elle conçut et fit construire par un atelier de mécanique à Douai ${ }^{61}$. Elle reçut un accueil bienveillant des académies de Lille et de Strasbourg. En 1931, elle élargit son action aux académies du grand Est (Picardie, Bourgogne, Champagne-Ardennes, Lorraine, Franche Comté .... $)^{62}$.

Tout s'enchaîna : conférence pour des aveugles en 1932, rencontres comme celle d'A. Richardot ${ }^{63} \mathrm{~d}$ 'astro-camping qui lui donna l'idée de bricoler de petites lunettes «bon marché » réalisées avec son collègue Réant à l'AAN à partir de tubes en carton et de manches à balais ${ }^{64}$; en 1934, ce fut la jonction avec l'École nouvelle et la Société Belge de Pédotechnie $^{65}$; en 1936, à Lille, elle donna des cours d'astronomie avec 1'Union Française de la Jeunesse, puis des émissions pour la Radiophonie scolaire $^{66}$. Chez les scouts et les éclaireurs, elle inséra la découverte du ciel dans leur approche de la nature ${ }^{67}$. Et puis elle intervint dans des lycées parisiens, des écoles normales, des athénées belges ainsi que des sanatoriums de la côte d'Opale et de Belgique, de Berck à Zuydcoote ${ }^{68}$. Elle projeta des films devant 800 élèves à Amiens et fonda un «brevet» pour animateurs en astronomie, suivi de sa conférence «Plein Ciel» à la séance inaugurale de la Société astronomique de Liège, le 3 décembre $1938^{69}$. Enfin, en 1939, elle incita les scouts à faire des feux de la SaintJean dans leurs camps au solstice d'été pour marquer par des observations l'apothéose de la lumière et pour relancer la Fête du Soleil instituée par Flammarion et Eiffel $^{70}$.

Elle connut la consécration en devenant secrétaire générale du Planétarium de Paris en 1937, chargée de coordonner la conception et l'animation de cet équipement, en service quai de la Seine, juste en face

${ }^{61}$ L'Astronomie, 1938, p. 412.

${ }^{62}$ L'Astronomie, 1937, p. 365.

${ }^{63}$ Ibid., p. 367.

${ }^{64}$ Ibid., p. 367.

${ }^{65}$ Ibid., p. 424.

${ }^{66}$ Ibid., p. 428.

${ }^{67}$ L'Astronomie, 1938, p. 411.

${ }^{68}$ Ibid., p. 414.

${ }^{69}$ Revue de la Société Astronomique de Liège à cette date.

${ }^{70}$ Joseph Leclerc, «Les fêtes du Soleil », L'Astronomie, 1940, pp. 156-158; JeanMichel Faidit, «La fête du Soleil », L'Astronomie, juin 2004, pp. 470-421 et juillet-août 2004, pp. 318-321. 
de la Tour Eiffel, durant les six mois de l'Exposition universelle de 1937. À raison de quinze séances chaque jour, de dix heures du matin à minuit, sa capacité de plus de 300 places permit à 800000 spectateurs de découvrir le ciel et les prouesses de la technologie moderne ${ }^{71}$. Un record jamais égalé !

L'année suivante, à la fin de l'hiver, nouvelle aventure. En mars, ce fut la création du Groupe Camille Flammarion des Éclaireurs de France au centre de Cappy avec l'appui d'Henri Lhote, jeune explorateur du Sahara. En quatre mois, ses membres, une dizaine de jeunes gens et jeunes filles réunis autour de Reysa, montèrent un «Stand-exposition / Planétarium » pour présenter l'astronomie lors de la fête du 26 juin au Jardin d'acclimatation. Quatre panneaux, d'une surface totale de 40 mètres carrés, et des appareils de démonstration de la voûte céleste concrétisèrent le hall d'exposition du grand planétarium de 1937 qui n'avait pu être réalisé entièrement dans les délais. Quatre causeries s'enchaînèrent tout au long de la journée, typiques des premières séances de planétarium (« Le ciel de ce soir », « Le Tour du Ciel en 365 jours », « Pourquoi sommes-nous en été ? » et «Vers la Croix du Sud et vers le pôle Nord»), alternées avec des séances de démonstration, le tout articulé autour d'une toile de tente itinérante. C'était un nouveau concept pour la diffusion de l'astronomie. Un millier de visiteurs furent accueillis, parmi lesquels se trouvait Jean Zay, ministre de l'Éducation nationale ${ }^{72}$.

Tout cela représentait beaucoup de travail et de voyages qui l'obligèrent à démissionner de son poste de secrétaire de l'AAN $^{73}$, mais c'était «Un investissement itinérant pour l'avenir», selon Reysa Bernson, dans son article dans la revue L'Astronomie, curieusement daté à la fin «septembre 1938 », qui est le mois des accords de Munich. Elle avait su susciter l'émerveillement de nombreux enfants devant cette immensité du ciel : «Et ce qui est difficile à exprimer, c'est l'élan qui jette les enfants vers ces révélations, le hurlement d'enthousiasme d'une classe à l'arrivée de l'« astronome », la joie tangible qui les anime tandis qu'ils écoutent, les yeux brillants - et le plaisir qu'on éprouve soi-même à l'avoir créée... ».

\footnotetext{
${ }_{71}^{71}$ Archives privées d'Armand Delsemme, communiquées par Hélène Louon.

${ }^{72}$ L'Astronomie, 1938, p. 412.

${ }^{73}$ Lettre adressée au président de l'AAN : « [...] faisant état de ses occupations passées au Planétarium de Paris et de ses occupations futures qui l'ont tenue et qui la tiendront éloignée de notre région assez fréquemment, elle estime ne plus pouvoir apporter une collaboration suffisamment active [...]», bulletin de l'AAN, 1938.
} 
Son absence dans les bulletins de L'Astronomie de l'après-guerre était peu compatible avec son dynamisme. À l'évidence, la guerre n'avait pas été sans dommages.

\section{Auschwitz, destination finale de deux femmes juives exceptionnelles}

En 1936, selon le recensement ${ }^{74}$, Dweira et Reysa Bernson résidaient encore ensemble à Lille, non plus boulevard de la Liberté mais à quelques centaines de mètres, 10 rue Baptiste Monnoyer. Curieusement Reysa est notée «chef du ménage » et «docteur » probablement en sciences et non en médecine. À 32 ans, elle aurait donc pris en charge sa mère, alors âgée de 65 ans, inscrite «sans profession ». Dweira, quant à elle, est toujours qualifiée de « Russe », ce qui signifierait qu'elle n'avait toujours pas la nationalité française et qu'elle se trouvait dans une situation dangereuse après la déclaration de guerre, en 1941, entre l'URSS communiste et l'Allemagne nazie : Russe et juive, elle était une cible privilégiée pour la déportation. Après 1936, la fin de la vie de Dweira et Reysa reste mal connue car leurs noms disparaissent des archives pour ne réapparaître que sur des listes de déportés.

Les deux noms «Bernson Dveira » et «Bernson Reysa » figurent sur la « liste des citoyens français arrêtés par les autorités allemandes pendant le mois de février $1944 »{ }^{75}$. Selon ce document, elles demeuraient alors « 15 rue St Thibault » à Dreux où elles furent arrêtées le 23 février 1944 au motif de leur origine juive, leurs fiches de signalement portent les $\mathrm{n}^{\circ} 379$ et 380 . Elles étaient encore, à cette date, connues sous le nom de Bernson et non de Verhaeghe, nom qui a une consonance moins suspecte d'appartenance au judaïsme.

Cette liste fut transmise à « Monsieur de Brinon, Ambassadeur de France, secrétaire d'État auprès du Chef du Gouvernement, Délégué Général du Gouvernement Français dans les Territoires occupés » par le préfet d'Eure-et-Loir ${ }^{76}$. Dès le 4 mars 1944, elle alimenta le « fichier de Brinon » dans lequel on retrouve la fiche de Reysa Bernson sur laquelle on lit qu'elle fut arrêtée le 23 février 1944 avec la mention : « JUIF $»{ }^{77}$.

\footnotetext{
${ }^{74}$ AML, recensement de 1936, 1 F 44.

${ }^{75}$ Archives des victimes des conflits contemporains à Caen (désormais DAVCC Caen), LA 11193.

${ }^{76}$ DAVCC Caen, AC-22P-3083.

${ }^{77}$ Ibid. Le fichier de Brinon rassemble les fiches émanant de la Délégation générale du Gouvernement dans les Territoires Occupés (DGTO), source DAVCC. Fernand de Brinon (1885-1947), avocat et journaliste, fut une grande figure de la Collaboration
} 
Après leur arrestation, leurs derniers jours sont comptés en petit nombre. Elles furent rapidement transférées à Drancy en passant par la maison d'arrêt de Chartres où elles furent enregistrées sous les numéros d'écrou 402 pour Reysa et 405 pour Dweira, le jour même de leur arrestation et cette fois sous le nom de Verhaeghe et non plus Bernson. Le document provenant du fonds de la maison d'arrêt précise qu'elles furent arrêtées par les autorités allemandes et transférées sur ordre des mêmes autorités qui les ont escortées jusqu'à Chartres. Le motif indiqué ici est: «passagère juive». Le registre des écrous ignore le lieu «sur lequel elles doivent être dirigées » mais elles sortirent de la maison d'arrêt le 24 février $^{78}$. Elles séjournèrent à peine 24 heures à Chartres et il est légitime de supposer que c'est le jour même de leur sortie qu'elles arrivèrent au camp de Drancy où elles furent internées seulement une dizaine de jours.

Bien que le lieu de détention ne soit pas précisé sur la liste d'arrestation, le Mémorial de la déportation des Juifs de France, établi par Serge Klarsfeld, renseigne quelque peu sur leurs derniers jours. Leurs noms se lisent encore sur la liste du convoi 69 qui quitta Drancy, où elles ont donc séjourné quelques jours, en direction d'Auschwitz le 7 mars 1944, toujours sous leur identité de Verhaeghe. Il ne semble pas qu'elles aient tenté de dissimuler leur judéité sous le nom bien flamand de leur mari et père puisqu'elles ont été inscrites, toutes deux, sous le nom de Bernson sur la liste d'arrestation d'Eure-et-Loir.

Le 8 mars 1944, alors que leur convoi 69 avait déjà quitté le camp de Drancy, leurs fiches furent réceptionnées par les services de de Brinon $^{79}$. Le classement administratif de leur arrestation prit plus de temps que leur déportation! «Ce convoi comprend 1501 personnes, dont 812 hommes et 689 femmes. Parmi eux 178 enfants de moins de 18

pendant toute la durée de la guerre. Bien que marié à une juive de Belgique, convertie au catholicisme, il fut Délégué général du Gouvernement de Vichy auprès des autorités militaires allemandes d'occupation, de 1940 à 1944, et il œuvra systématiquement à l'arrestation des juifs étrangers, comme l'était peut-être encore Dweira Bernson, et aussi français, comme l'était Reysa Bernson. À la Libération il fut condamné à mort et exécuté le 15 avril 1947. Voir Gilbert Joseph, Fernand de Brinon, l'aristocrate de la collaboration, Paris, Albin Michel, 2002 ; Pascal Ory, Les collaborateurs, Paris, éd. du Seuil, 1972, réédité en 1999.

${ }_{78}$ Archives départementales de l'Eure-et-Loir (désormais AD 28), 1055 W 27, fonds provenant de la maison d'arrêt de Chartres. Dans ce document, leur dernier lieu de séjour est localisé à Dreux et Dweira est née à Brest Finistère ! Nous remercions chaleureusement Christiane Riguet, archiviste, qui a bien voulu faire cette recherche à notre demande.

${ }^{79}$ DAVCC Caen, AC-22P-3083. 
ans [...] 110 hommes furent sélectionnés (Mles 174904 à 175 013) et 80 femmes environ. En 1945, 20 survivants, dont 5 femmes ${ }^{80}$. Le sort tragique des déportés de ce convoi est connu grâce au témoignage de Guy Kohen, paru dès 1945 immédiatement après son retour ${ }^{81}$. Né le 27 août 1924, Guy Kohen fut arrêté le 28 janvier 1944, parce que juif, et transféré à la prison de Limoges où il fut torturé car il refusait de donner l'adresse de son père. Le 24 février, le lendemain de l'arrestation de Dweira et Reysa Bernson, il fut dirigé, avec des codétenus, vers Drancy. Il y fut donc interné et déporté en même temps qu'elles, aussi la description qu'il donne du camp de Drancy, du transport et de l'arrivée au camp d'extermination nous renseigne-t-elle sur les derniers moments de la mère et très probablement de la fille.

L'atmosphère y [camp de Drancy] était étrange. Rien n'y était clair ; un grand mystère planait sur le sort qui nous était réservé [p. 33...] En arrivant il fallut remettre tout notre argent et nos bijoux. Nous subîmes une fouille très sérieuse [p. 34...] Puis vint une nouvelle. Le départ était fixé pour le 7 mars. Trois jours avant le transport, les barbelés furent mis devant la partie du camp réservée aux déportables. Pendant ces trois derniers jours, la nourriture nous fut moins parcimonieusement répartie [p. 35...]

Le 7 mars, donc, ayant reçu des vivres pour trois jours, nous fûmes chargés à soixante par wagon à bestiaux; hommes, femmes, enfants y étaient réunis [p. 36...]

Le volume des bagages empêchait que chacun pût s'étendre. Le manque de place et la promiscuité qui régnait forcément parmi nous (pas d'hygiène possible, une seule tinette pour tout le wagon, que nous vidions quand les SS nous en donnaient l'autorisation ; parfois, quand elle était trop pleine, nous étions obligés de nous retenir de longues heures) nous rendirent le voyage atroce [p. 36...]

Le 10 mars, au petit matin, nous arrivâmes à destination. Des coups brutaux furent frappés aux portes des wagons. L'ordre : «Préparez-vous à descendre », nous fut donné. Puis les portes s'ouvrirent et nous vîmes sur le quai quelques SS, très peu, et de nombreux bagnards [p. 39...]

Nous dûmes abandonner tous nos bagages sur le quai. Nous passions tous devant un SS, un très fort gaillard qui tenait une cravache à la main. De temps à autre, il arrêtait quelqu'un, lui posait une question puis l'envoyait à sa gauche où quelques hommes commençaient déjà à former un groupe [p. 39].

Guy Kohen, âgé de 19 ans, fut sélectionné pour le travail. Ni Dweira, ni Reysa n'ont survécu. Il est certain que Dweira, âgée de 73

\footnotetext{
${ }^{80}$ Serge Klarsfeld, Mémorial... op. cit. Présentation du convoi 69.

${ }^{81}$ L'ouvrage a été réédité : Retour d'Auschwitz. Souvenirs du déporté 174 949, Paris, éditions Le Manuscrit, 2006.
} 
ans, fut immédiatement dirigée vers la chambre à gaz. Reysa, âgée de 40 ans, a pu être épargnée en un premier temps ${ }^{82}$.

Toutefois, leur arrestation ne passa pas complètement inaperçue. Aux archives départementales d'Eure-et-Loir et du Nord, on peut découvrir un échange de lettres entre les préfets de ces deux départements et un couple : M. et Mme E. Malenge-Dorchies domiciliés au 53 boulevard de la Liberté à Lille, peu éloigné du 217 où habitaient Dweira et Reysa Bernson avant la guerre ${ }^{83}$. Entre le 18 février et le 17 mars 1944, ce M. Malenge rassembla des documents: extraits de naissance de Désiré Verhaeghe et de ses parents ainsi que l'extrait de baptême de Désiré Verhaeghe. Ils accompagnaient une lettre que Malenge adressa au préfet du Nord le 24 mars, ainsi formulée :

Monsieur le Préfet,

Nous avons l'honneur de vous demander respectueusement s'il serait possible, par votre intermédiaire, d'obtenir des nouvelles de nos parentes :

Madame VERHAEGHE-BERNSON

Mademoiselle Reysa VERHAEGHE, sa fille, arrêtées toutes deux à Dreux, vers la fin Février 1944.

Elles étaient respectueusement, l'épouse et la fille de feu, le Docteur Désiré VERHAEGHE, ancien Conseiller Général et adjoint au Maire de LILLE.

Nous ignorons totalement le motif de leur arrestation.

Lorsqu'il commença à rassembler ses documents, Dweira et Reysa n'étaient pas encore arrêtées. En revanche, lorsque le 24 mars il rédigea sa lettre tapée à la machine, elles étaient très probablement déjà décédées ! La lettre fut enregistrée à la préfecture du Nord le 3 avril. Par un courrier du 10 avril, le préfet transmit la requête de Malenge à son collègue d'Eure-et-Loir. La réponse du chef de cabinet de ce dernier,

\footnotetext{
${ }^{82}$ Nos démarches auprès des archives du Musée d'Auschwitz furent inutiles car les fichiers concernant ce convoi ont disparu. Dans une correspondance du 21 février 2017, il nous fut répondu: "Their names are mentioned only in the list of deportees from Drancy to Auschwitz of March 7, 1944. There is no information about their further fate ». Puis Danielle Delmaire a persévéré pour retrouver une trace de la mère et sa fille après leur arrivée dans le camp d'Auschwitz en demandant de l'aide au Service International d'Arolsen, en Allemagne. Un courriel du 20 mars apporta la même réponse : "There was no information to be found on their incaceration in Concentration Camp Auschwitz... many documents were lost or destroyed ». Nous remercions les services de ces deux centres d'archives pour l'aide apportée dans ces recherches, même si elles ont été vaines.

${ }^{83}$ Ces lettres qui se complètent se trouvent aux $\mathrm{AD} 28$ à la cote $14 \mathrm{~W} 49$ et aux $\mathrm{AD} 59$ à la cote $1 \mathrm{~W} 1879$.
} 
datée du 27 avril et arrivée en préfecture du Nord le 2 mai, est tragiquement laconique :

[...] j'ai l'honneur de vous faire connaître que Mme et Melle VERHAEGHE ont été arrêtées par les Autorités Allemandes le 23 février 1944 en même temps que 54 autres personnes d'origine juive.

Les intéressées auraient été emmenées à la prison de Chartres et dirigées ensuite sur le camp de Drancy.

Les services de la préfecture d'Eure-et-Loir sont formels sur la raison de l'arrestation : « l'origine juive », mais semblent dubitatifs quant à la suite donnée à cette arrestation. Pourtant, ce fut bien l'itinéraire de Dweira et Reysa avant d'embarquer pour Auschwitz. Enfin, le 17 mai 1944, le préfet du Nord communiqua la réponse à Malenge.

La démarche de Malenge-Dorchies est étonnante. Il présenta Dweira et Reysa Bernson-Verhaeghe comme ses parentes mais sans préciser le degré. La parenté ne pouvait être que du côté de Désiré Verhaeghe, c'est de cette famille qu'il a fourni des actes d'état civil, car Dweira Bernson n'avait pas de famille en France. En outre, il savait où trouver ces actes d'état civil : à Hazebouck. Il semble bien connaître la famille Verhaeghe. Se doutait-il que Dweira et Reysa couraient un danger en tant que juives? On peut le supposer puisqu'il ajouta aux extraits de naissance l'extrait de baptême de Désiré Verhaeghe. C'était une preuve, au moins pour Reysa, que les Verhaeghe n'appartenaient pas à la « race juive » comme s'exprimaient les nazis et leurs complices. Et Malenge n'a pas manqué de mentionner l'honorabilité de Désiré Verhaeghe pour appuyer sa demande. Sans doute espérait-il accélérer le processus de recherche et éventuellement obtenir une certaine clémence de la part des responsables de l'arrestation des juifs.

On peut aussi s'étonner de sa démarche en considération des risques qu'il prit, s'il en était conscient. S'il savait que Dweira et Reysa étaient juives - il devait le savoir s'il était un parent de Désiré Verhaeghe et c'est sans doute la raison pour laquelle il a fourni un extrait de baptême de celui-ci - il prenait le risque de faire porter sur lui-même une suspicion d' « appartenance à la race juive ». Il semblait donc très inquiet quant au sort de ses parentes.

On peut encore s'étonner de l'absence de cette suspicion de la part du préfet du Nord qui accepta de donner suite à la requête de Malenge. Il aurait pu classer l'affaire voire inquiéter Malenge. En fait, le préfet Carles en poste depuis 1936, s'il se montra très anticommuniste, fut 
laxiste envers les juifs. Sa femme était d'origine juive et aida un réseau clandestin de secours aux juifs ${ }^{84}$.

L'échange de lettres s'arrêta en mai 1944 et il était trop tard pour tenter de sauver Dweira et Reysa Bernson. Au lendemain de la guerre, Malenge-Dorchies habitait toujours au 53 boulevard de la Liberté à Lille selon l'annuaire Ravet-Anceau de 1947.

Pendant la guerre, un couple lillois les rechercha et après la guerre l'astronome lilloise Reysa Bernson ne fut pas complètement oubliée. L'Association Astronomique du Nord (AAN) existait toujours. En 1955, son bureau sollicita l'Université catholique de Lille, qui possédait un observatoire, pour qu'elle accepte de recevoir un instrument d'observation et du matériel de laboratoire que l'association ne pouvait plus entretenir. Elle ne possédait qu'une cabane qui les abritait mal. Pour convaincre le recteur de l'université, le président de l'AAN rappela son passé qui devait : «[...] être garant de son avenir, outre les pionniers dont M. le Chanoine STOFFAES, je citerai entre autres, Melle BERNSON [...] ${ }^{85}$. Deux autres noms seulement suivent celui de Reysa tandis que Henri D'Halluin était toujours secrétaire de l'association.

Des recherches concernant Reysa Bernson, après la guerre, se sont avérées vaines. En effet, le 3 août 1946, le directeur de l'Observatoire de Paris écrivit une lettre au ministre des Anciens combattants et victimes de la guerre (service des déportés) afin de s'assurer du décès de Reysa Bernson, qu'il nommait, par erreur, Rery ou Rera. «Cette jeune fille, précise-t-il, d'origine israélite a dû, je suppose, être déportée en Allemagne pendant la guerre. Mlle Bernson ayant déposé différents objets à l'Observatoire de Paris pendant la guerre, je serais heureux de pouvoir remettre ceux-ci aux héritiers, s'il en existe ${ }^{86}$. Ce directeur, deux ans après la Libération et plus d'un an après la fin de la guerre, ignorait la destination des déportés juifs, qu'il situait en Allemagne. Le nom d'Auschwitz commençait tout juste à être connu.

\footnotetext{
${ }^{84}$ Lire Monique Heddebaut, « Sans armes face à la rafle du 11 septembre 1942 (dans la « Zone rattachée » à Bruxelles), Tsafon, revue d'études juives du Nord, $\mathrm{n}^{\circ} 70$, automne 2015 - hiver 2016, pp. 119-169. Cet article a aussi été publié à part.

${ }^{85}$ Archives de l'Université catholique de Lille. BF, rapports de nature scientifique, $5 \mathrm{c}$ Association Astronomique du Nord, lettre du 25 mai 1955. Voir aussi Marie-Thérèse Pourprix, Des mathématiciens à la faculté des sciences de Lille 1854-1971, Paris, L'Harmattan, 2004, p. 95: «L'Observatoire [de la faculté de Physique] a subi des dégâts sans gravité. M. D'Halluin en est, quelques temps, le directeur intérimaire », en 1942, et les enseignants ayant repris leurs fonctions, l'astronomie était de nouveau enseignée. Toutefois, Reysa Bernson était absente !

${ }^{86}$ DAVCC Caen, 21 P 424741.
} 
Que s'est-il passé entre 1940 et février 1944 ? Quand Dweira et Reysa Bernson ont-elles quitté Lille et quand se sont-elles installées à Dreux ? Et pourquoi se réfugier à Dreux ? À ce jour, aucune réponse ne peut être apportée à ces questions. Toutefois, la lettre du directeur de l'Observatoire permet de supposer que Reysa Bernson avait éventuellement souhaité sauvegarder son matériel d'astronome en le déposant en lieu sûr à l'Observatoire de Paris et ceci «pendant la guerre », avec l'espoir de le retrouver ${ }^{87}$.

\section{En guise de conclusion}

La vie de Dweira Bernson commença dans les crimes des pogromes en Russie et se termina dans les crimes contre l'humanité à Auschwitz. Dans l'un et l'autre cas, sa faute était d'être née juive. Elle a voulu oublier sa judéité et s'adonner à l'amélioration de la condition ouvrière, quelle que fût cette population misérable. Les antisémites, grâce à une législation criminelle, la condamnèrent à mort, lui rappelant sa judéité. Sa vie commença en Biélorussie et se termina en Pologne. Entretemps elle s'était déroulée en France, dans la région industrielle du Nord. Actuellement, cette région ne conserve aucun souvenir d'elle, si ce n'est par son mari.

Quant à sa fille Reysa Bernson, elle subit la même fin de vie tragique alors qu'elle était «demi-juive », « fille d'aryen ». Une grande complicité semble unir les deux femmes: elles cohabitaient constamment, la fille portait le prénom de sa grand-mère maternelle et se fit connaître comme astronome renommée sous le patronyme de sa mère et jamais sous celui de son père. Elle aussi est oubliée dans sa ville natale. Les journaux régionaux la citèrent uniquement lors de l'inauguration de l'école de plein air fondée par son père et qui en porte le nom.

En fait, les seules traces bien visibles qu'elles laissent sont celles de leur mort : deux noms dans le Mémorial de la Déportation des Juifs de France de Serge Klarsfeld et deux noms sur le mur des déportés du

\footnotetext{
${ }^{87}$ Sollicitées par Jean-Michel Faidit, Marie-Agnès Dubos, Suzanne Débarbat et Émilie Kaftan de l'Observatoire de Paris ont vainement cherché dans les archives de cet établissement. Il semble probable que Reysa Bernson fut ponctuellement employée comme jeune astronome pour des missions d'observation, ce qui expliquerait, après son investissement au Planétarium durant l'année 1937, qu'elle ait démissionné du secrétariat de l'Association Astronomique du Nord puis qu'elle ait déposé du matériel à l'Observatoire, mais quand se serait-elle installée à Dreux, près de Paris ?
} 
Mémorial de la Shoah à Paris, alors qu'elles n'auraient pas dû être déportées puisque l'une était épouse d'《 aryen » et l'autre « demi juive ». Rien n'est dit sur leurs vies si fructueuses et bénéfiques pour les femmes et pour les enfants, à la différence de leur mari et père qui bénéficie d'une trace plus glorieuse : le nom d'une rue à Lille.

Une plaque sur leur lieu de résidence, sinon leur nom attribué à un établissement scolaire ou à une salle des facultés de médecine ou de pharmacie de Lille, entretiendrait leur souvenir. 
Thèse de doctorat en médecine de Dweira Bernson

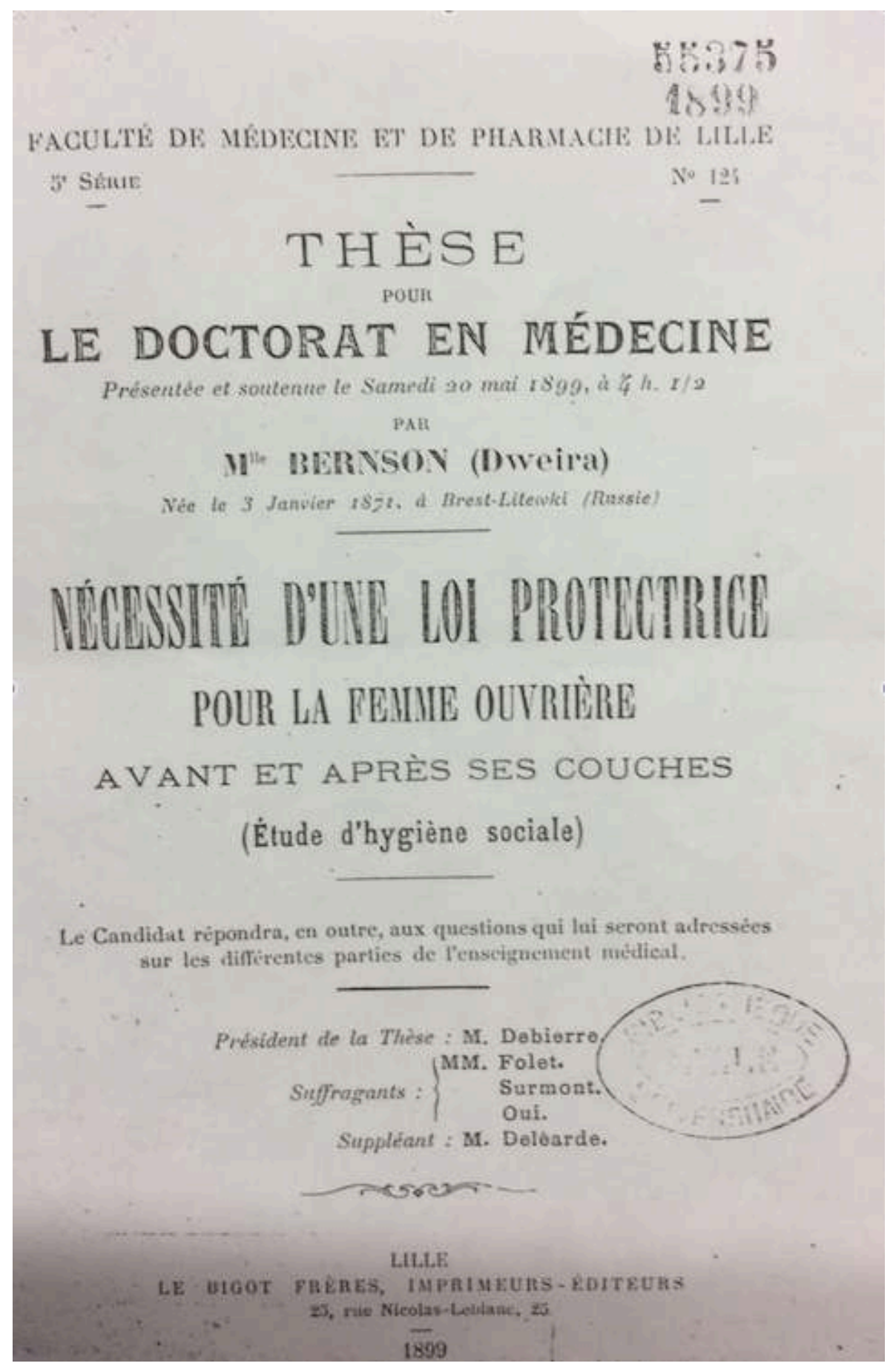

Source : AML 1 D 4, dossier Désiré Verhaeghe 
Tsafon 74 : Vie et mort de deux femmes juives. À l'ombre d'un mari et d'un père

Thèse de doctorat en médecine de Désiré Verhaeghe

Page de dédicaces

\section{A MES PARENTS}

qui n'ont jamais épargaé ni les saerilices, ni les dévouements.

\section{A MON AMHe LE Docteun DÉmorah BERNSON}

\section{A tous ceux, Maithes et Camarades}

qui m'ont fait comprendre la Seience et la Vie, et ont diéveloppé en moi le desir et la volonté d'un continuel progries.

\section{A mes Fréres de Prolétartat manuel}

qui n'ont pas eu le bonlseur de pouvoir s'approcher de la source léconde de Vérité et de Reauté; a qui je suis redevable des loisirs de ma jeunesse privilégiée: devant qui je tue déclare responsable de l'emploi que j'en ai fait, de l'usage que je terai dans l'avenir du savoir acquis......

Source : AML 1 D 4, dossier Désiré Verhaeghe 


\section{Conférences et interventions scolaires de Reysa Bernson}

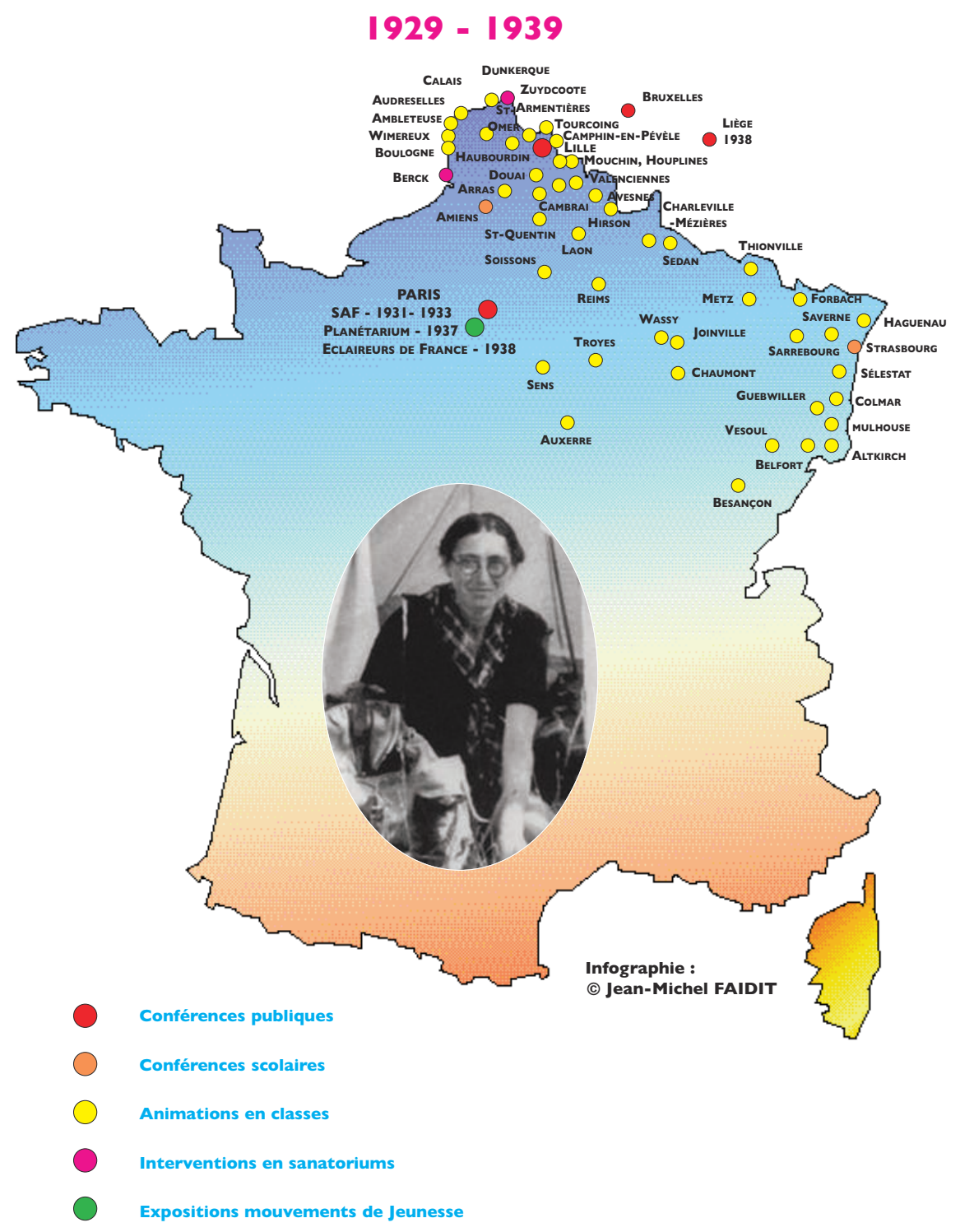

Interventions scolaires de Reysa Bernson dans le quart Nord-Est de la France (1929-1939)

Infographie J-M. Faidit

Photo : Archives de l'AAN 